\title{
Supporting Information: Unravelling Local Structure of Molten Salts via X-Ray Scattering, Raman Spectroscopy, and Ab Initio Molecular Dynamics
}

\author{
Santanu Roy, ${ }^{1}$ Martin Brehm, ${ }^{2}$ Shobha Sharma,${ }^{3}$ Fei Wu, ${ }^{3}$ Dmitry S. Maltsev, ${ }^{4}$ Phillip Halstenberg, ${ }^{1,4}$ \\ Leighanne C. Gallington, ${ }^{5}$ Shannon M. Mahurin, ${ }^{1}$ Sheng Dai, ${ }^{1,4}$ Alexander S. Ivanov, ${ }^{1 *}$ Claudio J. \\ Margulis, ${ }^{3 *}$ Vyacheslav S. Bryantsev ${ }^{1 *}$ \\ AUTHOR ADDRESS. \\ 1 Chemical Sciences Division, Oak Ridge National Laboratory, Oak Ridge, Tennessee 37831, United States \\ 2 Institut für Chemie, Martin-Luther-Universität Halle-Wittenberg, Von-Danckelmann-Platz 4, 06120 Halle (Saale), Germany \\ 3 Department of Chemistry, University of Iowa, Iowa City, Iowa 52242, United States \\ 4 Department of Chemistry, University of Tennessee, Knoxville, Tennessee 37996, United States \\ 5 X-ray Science Division, Advanced Photon Source, Argonne National Laboratory, Argonne, Illinois 60439, United States \\ *Vyacheslav S. Bryantsev, Email: bryantsevv@ornl.gov \\ * Alexander S. Ivanov, Email: ivanova@ornl.gov \\ * Claudio J. Margulis, Email: claudio-margulis@uiowa.edu
}

\section{Sample preparation}

Magnesium chloride $\left(\mathrm{MgCl}_{2}\right)$ is a highly hygroscopic compound and the salt hydrate $\left(\mathrm{MgCl}_{2} \bullet \mathrm{nH}_{2} \mathrm{O}\right)$ readily undergoes hydrolysis at elevated temperatures, leading to the formation of $\mathrm{MgOHCl}$ above $\sim 300{ }^{\circ} \mathrm{C}$ and $\mathrm{MgO}$ above $\sim 5500^{\circ} \mathrm{C} .{ }^{1,2}$ For our X-ray diffraction and Raman measurements the $\mathrm{MgCl}_{2}$ (anhydrous, $>98 \%$ ) was purchased from Sigma-Aldrich and then additionally purified via fractional distillation as described in our previous studies. ${ }^{3}$ The distillation process was needed to insure that the resulting anhydrous $\mathrm{MgCl}_{2}$ does not contain any measurable amounts of the corresponding hydroxychlorides or oxides. After the distillation, the $\mathrm{MgCl}_{2}$ was transported into an Ar-filled glovebox without exposure to air and kept in the glovebox. The obtained salt was then crushed to fine powder inside of the glovebox, added to a thin-walled quartz capillary (Charles Supper Co., 2.0 mm O.D., 0.010 $\mathrm{mm}$ wall thickness), evacuated to a pressure of $1.0 \times 10^{-3} \mathrm{Torr}$, and flame sealed. To prepare $\mathrm{KCl}-\mathrm{MgCl}_{2}$ salt mixture, proper masses of dry $\mathrm{KCl}$ (99.999\% Suprapur reagent grade, sealed ampoule) and $\mathrm{MgCl}_{2}$ were ground and mixed together with a mortar and pestile to obtain a 50:50 molar ratio. The mixture was then fused together under dynamic vacuum, transferred to the glovebox, and crushed to fine powder.

\section{High-energy X-Ray scattering measurements}

High-energy X-ray scattering experiments on molten $\mathrm{MgCl}_{2}$ at $1123 \mathrm{~K}$ were made using the beamline 11 -ID-B at the Advanced Photon Source (APS) with an incident X-ray wavelength of $0.2115 \AA$ (energy $=58.6 \mathrm{keV}$ ). The diffracted X-rays were collected using an amorphous silicon-based area detector (Perkin Elmer XRD1621) with $200 \times 200$ micron pixels. The detector was placed $170 \mathrm{~mm}$ behind the sample, giving an accessible q-range of $0.5-$ $24.5 \AA$. Calibration of the precise sample to detector distance, detector tilt and rotation, and beam center was performed in the GSAS II program ${ }^{4}$ using crystalline $\mathrm{CeO}_{2}$ powder as the standard. Diffraction patterns were collected for the molten $\mathrm{MgCl}_{2}$ sample in a $2.0 \mathrm{~mm}$ quartz capillary (Charles Supper Co., $0.010 \mathrm{~mm}$ wall thickness) in the furnace and for an empty capillary in the furnace at the same temperature. GSAS II was used to integrate the obtained 2D detector images to 1D diffraction patterns. Masks were applied to remove dead pixels and the beamstop from data integration. PDFgetX $2^{5}$ was then used to subtract the background signal (empty quartz capillary and air scattering) and to perform additional corrections (sample self-absorption, multiple scattering, and inelastic 
Compton scattering) following standard procedures. ${ }^{6,7}$ The obtained data were subsequently normalized with respect to the average electron density given by the weighted sum of the ionic X-ray form factors for $\mathrm{Mg}^{2+} \mathrm{and}^{-}$ , yielding the total structure function, $\mathrm{S}(\mathrm{q})$ :

$S(q)=\frac{I_{\operatorname{coh}}(q)-\sum_{i} x_{i} f_{i}^{2}(q)}{\left[\sum_{i} x_{i} f_{i}(q)\right]^{2}}$

where $I_{c o h}$ is coherent scattering intensity, $x_{i}$ and $f_{i}(\mathrm{q})$ are the molar fraction and q-dependent X-ray ionic form factor of species $i$, respectively, and $\mathrm{q}$ denotes the magnitude of the scattering vector $(\mathrm{q}=4 \pi \sin (\theta) / \lambda)$, where $2 \theta$ is the scattering angle, and $\lambda$ is the incident X-ray wavelength. For the X-ray scattering experiments a versatile hightemperature cell originally designed by Chupas et al. (ref. 65 in the main text) was used at the Advanced Photon Source. Although in this setup a sheathed K-type thermocouple with small $\left(0.010^{\prime \prime}\right)$ diameter was used to minimize heat transfer, we still observed some temperature variations with an estimated error of $\pm 25 \mathrm{~K}$. Thus, to ensure complete melting of the sample the experiments were conducted at elevated temperature. Based on our previous experimental and computational findings, only small changes in the structure functions with respect to peak intensities can be observed within $\sim 100 \mathrm{~K}$ temperature range.

\section{Raman spectroscopy measurements}

High-temperature Raman measurements were carried out using a Horiba T64000 spectrometer. A diode laser of 532 $\mathrm{nm}$ was focused through a 50x objective of microscope. The power of the laser was $100 \mathrm{~mW}$. A Linkam heating stage TMS1100 depicted in Figure S1 was used for measuring Raman spectra of molten $\mathrm{MgCl}_{2}$ and $\mathrm{KCl}-\mathrm{MgCl}_{2}$ at $1073 \mathrm{~K}$. Unlike fluorides, chloride molten salts are not corrosive to glass, thus conventional optical window materials were used without any modifications. The stage was connected to a nitrogen flow $(15 \mathrm{ml} / \mathrm{min})$ to keep the samples $(\sim 50 \mathrm{mg})$ in alumina crucibles under an inert atmosphere. The total acquisition time was $10 \mathrm{~s}$ for each sample. The Raman shift was measured from 0 to $1200 \mathrm{~cm}^{-1}$ in the range that should cover the expected Raman signal of $\mathrm{MgCl}_{2}$ and $\mathrm{MgCl}_{2}-\mathrm{KCl}$ molten salts. ${ }^{8,9}$ Initially, to test our high-temperature Raman setup, we performed our measurements for the anhydrous $\mathrm{ZnCl}_{2}$ salt at room temperature and $633 \mathrm{~K}$. The obtained Raman spectra for $\mathrm{ZnCl}_{2}$ shown in Figure S2 successfully reproduced the Raman data reported by Alsayoud et al. ${ }^{10}$

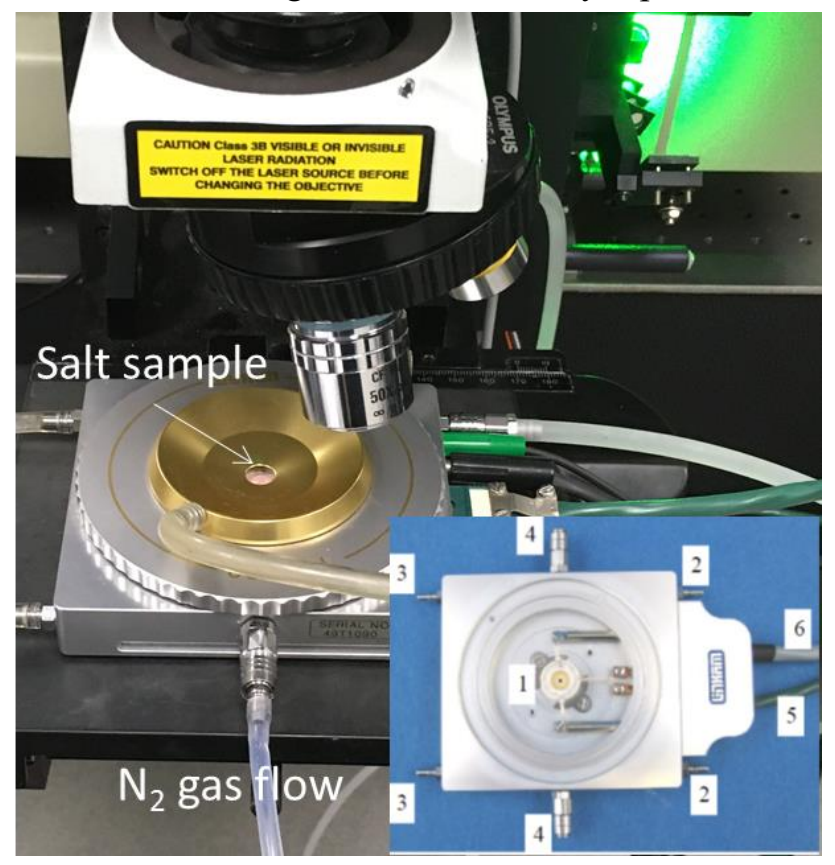

Figure S1. The high-temperature Raman setup used to investigate the salt samples. The inset shows the inside view of a Linkam heating stage: 1) alumina heating crucible, 2) water cooling connector, 3) bypass water cooling connector, 4) gas connector, 5) temperature sensor cable, 6) stage cable. 


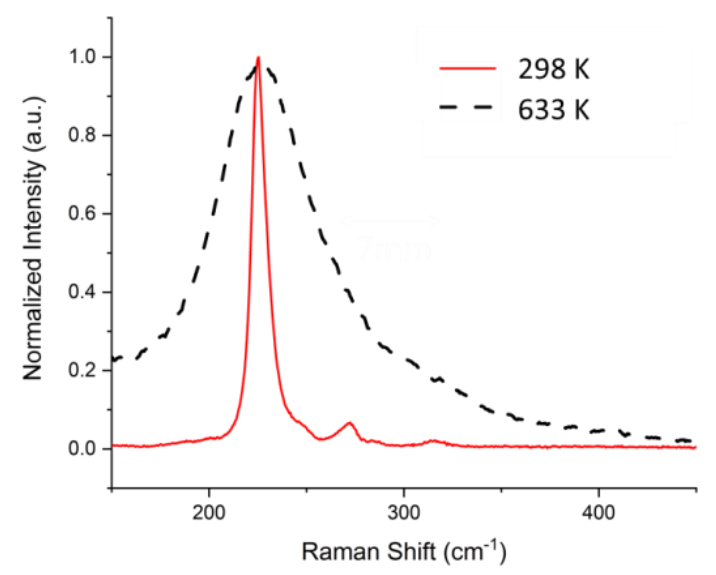

Figure S2. Unpolarized Raman spectra for $\mathrm{ZnCl}_{2}$ at 298 and $633 \mathrm{~K}$.

\section{Structure function calculations}

The total X-ray structure function, $\mathrm{S}(\mathrm{q})$ was calculated as

$S(q)=\frac{\rho_{0} \sum_{i} \sum_{j \geq i} x_{i} x_{j} f_{i}(q) f_{j}(q) \int_{0}^{\infty} 4 \pi r^{2}\left(g_{i j}(r)-1\right) \frac{\sin (q r)}{q r} d r}{\left[\sum_{i} x_{i} f_{i}(q)\right]^{2}}(1)$,

where $\rho_{0}$ is the number density, $g_{i j}(r)$ is the radial distribution function, and $x_{i}$ and $x_{j}$ are the mol fractions for ionic components $i$ and $j ; f_{i}(q)$ and $f_{j}(q)$ are the corresponding X-ray form factors for the ions. ${ }^{3,11}$

Partial $S(q)$ components are defined as

$S_{i j}(q)=2 \times \frac{\rho_{0} x_{i} x_{j} f_{i}(q) f_{j}(q) \int_{0}^{\infty} 4 \pi r^{2}\left(g_{i j}(r)-1\right) \frac{\sin (q r)}{q r} d r}{\left[\sum_{i} x_{i} f_{i}(q)\right]^{2}}$

and

$$
S_{i i}(q)=\frac{\rho_{0} x_{i} x_{i} f_{i}(q) f_{i}(q) \int_{0}^{\infty} 4 \pi r^{2}\left(g_{i i}(r)-1\right) \frac{\sin (q r)}{q r} d r}{\left[\sum_{i} x_{i} f_{i}(q)\right]^{2}} \text { (3). }
$$

For neutron scattering structure functions equations are identical except that the $q$-dependent X-ray form factors are replaced by the $q$-independent coherent neutron scattering lengths for the ions. ${ }^{12}$

\section{Raman spectra calculations}

To compute Raman spectra from the AIMD simulations, the well-known time-correlation formalism was applied. ${ }^{13}$, ${ }^{14}$ As the systems investigated consist of monoatomic ions rather than molecules, it was not possible to consider molecular quantities (as is otherwise often done), and the total simulation cell was considered instead. The polarizability tensor $\alpha$ of the simulation cell in each step was computed via finite differences by applying external electric fields. Snapshots of the simulation trajectory were re-computed with external homogeneous electric fields in $\mathrm{X}, \mathrm{Y}$, and $\mathrm{Z}$ direction (the applied field strength is 0.005 atomic units), and the entries of the polarizability tensor were obtained by observing the change in total cell dipole moment (three entries per external field direction), which was obtained via the Berry phase formalism as implemented in $\mathrm{CP} 2 \mathrm{~K}$. Based on the time series of the polarizability tensor, the TRAVIS program package ${ }^{15,16}$ was used to compute the Raman spectra.

From the time series of the polarizability tensor $\alpha(t)$ along the trajectory, the two invariants of the Raman spectrum can be computed via cross-correlation and subsequent Fourier transformations. ${ }^{14}$ These invariants are the isotropic contribution, defined as 


$$
\mathrm{a}(\omega):=\int_{-\infty}^{\infty}<\frac{\alpha_{\mathrm{xx}}^{\cdot}(\tau)+\alpha_{\mathrm{yy}}(\tau)+\alpha_{\mathrm{zz}}(\tau)}{3} \cdot \frac{\alpha_{\mathrm{xx}}^{\cdot}(\tau+\mathrm{t})+\alpha_{\mathrm{yy}}^{\cdot}(\tau+\mathrm{t})+\alpha_{\mathrm{zz}}^{\cdot}(\tau+\mathrm{t})}{3}>_{\tau} \exp (-\mathrm{i} \omega \mathrm{t}) \mathrm{dt}
$$

and the anisotropic contribution, defined as

$$
\begin{aligned}
\gamma(\omega):=\int_{-\infty}^{\infty}\left[\frac{1}{2}<\right. & \left(\alpha_{\mathrm{xx}}^{\cdot}(\tau)-\alpha_{\mathrm{yy}}^{\cdot}(\tau)\right) \cdot\left(\alpha_{\mathrm{xx}}^{\cdot}(\tau+\mathrm{t})-\alpha_{\mathrm{yy}}^{\cdot}(\tau+\mathrm{t})\right)>_{\tau} \\
& +\frac{1}{2}<\left(\alpha_{\mathrm{yy}}^{\cdot}(\tau)-\alpha_{\mathrm{zz}}(\tau)\right) \cdot\left(\alpha_{\mathrm{yy}}(\tau+\mathrm{t})-\alpha_{\mathrm{zz}}(\tau+\mathrm{t})\right)>_{\tau} \\
& +\frac{1}{2}<\left(\alpha_{\mathrm{zz}}^{\cdot}(\tau)-\alpha_{\mathrm{xx}}(\tau)\right) \cdot\left(\alpha_{\mathrm{zz}}(\tau+\mathrm{t})-\alpha_{\mathrm{xx}}(\tau+\mathrm{t})\right)>_{\tau}+\frac{3}{2}<\alpha_{\mathrm{xy}}(\tau) \cdot \alpha_{\mathrm{xy}}(\tau+\mathrm{t}) 2>_{\tau} \\
& \left.+\frac{3}{2}<\alpha_{\mathrm{yz}}(\tau) \cdot \alpha_{\mathrm{yz}}(\tau+\mathrm{t})>_{\tau}+\frac{3}{2}<\alpha_{\mathrm{zx}}(\tau) \cdot \alpha_{\mathrm{zx}}(\tau+\mathrm{t})>_{\tau}\right] \cdot \exp (-\mathrm{i} \omega \mathrm{t}),
\end{aligned}
$$

where $\alpha_{x x}$ denotes the time derivative of the XX component of the polarizability tensor and $\langle\cdot\rangle_{\tau}$ stands for the time average over the simulation time $\tau$.

Based on these invariants, the Raman intensities for a scattering angle of $90^{\circ}$ can be computed for different polarizabilities. ${ }^{14,17}$ If the polarization planes of incident light and observed signal are parallel ("parallel Raman spectrum"), the intensity is given by

$$
I_{\|}(\omega)=\frac{\hbar}{64 \pi^{3} \epsilon_{0}^{2} c^{4} k_{\mathrm{B}} T} \cdot \frac{\left(\omega_{\text {in }}-\omega\right)^{4}}{\omega} \cdot \frac{1}{1-\exp \left(-\frac{\hbar \omega}{k_{\mathrm{B}} T}\right)} \cdot \frac{1}{45}(45 a(\omega)+4 \gamma(\omega)) .
$$

If the polarization planes of incident light and observed signal are perpendicular to each other ("orthogonal Raman spectrum"'), the intensity is obtained as

$$
I_{\perp}(\omega)=\frac{\hbar}{64 \pi^{3} \epsilon_{0}^{2} c^{4} k_{\mathrm{B}} T} \cdot \frac{\left(\omega_{\text {in }}-\omega\right)^{4}}{\omega} \cdot \frac{1}{1-\exp \left(-\frac{\hbar \omega}{k_{\mathrm{B}} T}\right)} \cdot \frac{1}{45}(3 \gamma(\omega)) .
$$

If the polarization plane of the scattered light is ignored and all scattered light is considered ("unpolarized Raman spectrum"), the intensity is simply expressed as the sum of $I_{\|}(\omega)$ and $I_{\perp}(\omega)$ :

$$
I(\omega)=\frac{\hbar}{64 \pi^{3} \epsilon_{0}^{2} c^{4} k_{\mathrm{B}} T} \cdot \frac{\left(\omega_{\text {in }}-\omega\right)^{4}}{\omega} \cdot \frac{1}{1-\exp \left(-\frac{\hbar \omega}{k_{\mathrm{B}} T}\right)} \cdot \frac{1}{45}(45 a(\omega)+7 \gamma(\omega)) .(8)
$$

Due to the lengthy pre-factor, the intensity $I$ is obtained in the correct physical units of length. ${ }^{2}$ In all three cases, $\omega$ is the angular frequency of the signal, $\omega_{\text {in }}$ is the angular frequency of the incident laser light, $T$ denotes the temperature at which the observation is performed, $k_{\mathrm{B}}$ is Boltzmann's constant, $\epsilon_{0}$ stands for the vacuum permittivity, and $c$ denotes the speed of light in vacuum.

Finally, the depolarization ratio of the Raman spectrum can be computed as the quotient of the orthogonal and parallel Raman spectrum via

$$
\rho(\omega)=\frac{I_{\perp}(\omega)}{I_{\|}(\omega)}
$$

To increase the quality of the computed spectra, some well-known techniques from signal processing were utilized in the Fourier transformations in Equations 4 and 5, such as zero padding (increasing the length of the time series by a factor of 4 prior to the Fourier transformation) and application of a Hann window function $\left(\cos ^{2}\right)$. Other techniques that were applied here are less well-known, such as the finite difference correction and the increase of sampling quality via time reversibility of the simulation ("commutator trick"). For a description of these approaches, see Section V.A of the recent article on TRAVIS. ${ }^{16}$ 


\section{Different types of coordination/connectivity numbers and free energy calculations}

6.1 Coordination. If $r_{i}$ is the distance between the $i^{\text {th }} \mathrm{Cl}^{-}$and a cation and $r^{\dagger}$ represents the location of the boundary of the first chloride coordination shell determined from the first minimum of the cation-Cl $g(r)$, the coordination number of the cation is obtained by using a smooth function, $f_{i},\left(0<f_{i}<1\right)$ :

$$
n_{\mathrm{Cl}}=\sum_{i=1}^{N_{\mathrm{Cl}}} \frac{1-\left(\frac{r_{i}}{r^{\dagger}}\right)^{12}}{1-\left(\frac{r_{i}}{r^{\dagger}}\right)^{24}}=\sum_{i=1}^{N_{\mathrm{Cl}}} f_{i}
$$

Equation 10 allows smooth transitions of $\mathrm{Cl}^{-}$across the boundary of the first chloride solvation shell, providing the instantaneous value of the coordination number. $N_{\mathrm{Cl}}$ is the total number of $\mathrm{Cl}^{-}$ions. It should be noted that the choice of the form with specific powers $(12,24)$ in Equation 10 was specifically made to obtain continuous dynamics along the coordination number. Changing to very low powers $(6,12)$ can significantly overestimate the coordination number, but changing to higher powers $((16,32)$ or $(24,48)$ or $(48,96))$ will make systematic small changes in coordination number (see Figure S15), while the dynamics may be less smooth. To determine the population of a coordination state, we assume that the entire coordination number space is composed of four types of coordination numbers, namely $n=3, n=4, n=5$, and $n=6$. Then the population for each of these states were calculated respectively by defining the ranges $2.5<\mathrm{n}_{\mathrm{Cl}}<3.5,3.5<\mathrm{n}_{\mathrm{Cl}}<4.5,4.5<\mathrm{n}_{\mathrm{Cl}}<5.5$, and $5.5<\mathrm{n}_{\mathrm{Cl}}<6.5$. Note that, while integrating the RDF $(\mathrm{g}(\mathrm{r}))$ provides average coordination number, Equation 10 helps to understand the heterogeneity of coordination structures - revealing information about multiple stable/metastable coordination states that may exist.

6.2 Number of shared $\mathrm{Cl}^{-} . f_{i}$ in Equation 10 is the contribution of the $i^{\text {th }} \mathrm{Cl}^{-}$to the coordination structure of a cation. Thus, $f_{i j}$ and $f_{i k}$ can be treated as the contributions of the $i^{\text {th }} \mathrm{Cl}^{-}$respectively to the coordination structures of the $j^{\text {th }}$ and $k^{\text {th }}$ cations. Therefore, the number of $\mathrm{Cl}^{-}$ions shared between these two metals (M) cations is given by:

$$
n_{\mathrm{M}}^{\mathrm{Cl}}=\sum_{i=1}^{N_{\mathrm{Cl}}} f_{i j} f_{i k}
$$

In Equation 11, $n_{M}^{C l}$ is maximum for $\mathrm{Cl}^{-}$ions located in between two cations, i.e., the largest contribution comes from the shared overlapping coordination shells. Equation 11 can also be used to compute the number of cations coordinating with two different cations through shared chloride ions $\left(n_{\mathrm{M}}^{\mathrm{M}}\right)$-in this case, $f$ is computed using $r^{\dagger}$ obtained from the first minimum of the cation-cation $g(r)$.

6.3 Total connectivity number. We define the total cation-anion connectivity (coordination) number by modifying Equation 10 further:

$$
n_{\mathrm{Tot}}^{\mathrm{M}-\mathrm{Cl}}=\sum_{j=1}^{N_{\mathrm{M}}} \sum_{i=1}^{N_{\mathrm{Cl}}} \frac{1-\left(\frac{r_{i}}{r^{\dagger}}\right)^{12}}{1-\left(\frac{r_{i}}{r^{\dagger}}\right)^{24}} .
$$

Here, $N_{\mathrm{M}}$ is the total number of cations. Equation 12 is essentially the sum of the coordination numbers of all the cations. Similarly, the total cation-cation connectivity number is given by:

$$
n_{\mathrm{Tot}}^{\mathrm{M}-\mathrm{M}}=\sum_{j=1}^{N_{\mathrm{M}}-1} \sum_{i=j+1}^{N_{\mathrm{M}}} \frac{1-\left(\frac{r_{i}}{r^{\dagger}}\right)^{12}}{1-\left(\frac{r_{i}}{r^{\dagger}}\right)^{24}} .
$$

Here, $r_{i}$ is the distance of the $\mathrm{i}^{\text {th }}$ cation from the $\mathrm{j}^{\text {th }}$ cation and $r^{\dagger}$ is the location of the first minimum of the cationcation $g(r)$. The summations in Equation 13 ensure that the connectivity in a particular cation-cation pair is 
examined once. As an alternative to the total cation-anion and cation-cation connectivity, the total number of chloride ions shared between cations (considering all the cations) can provide further insight into connectivity and is determined as:

$$
n_{\mathrm{Tot}}^{\text {Shared }}=\sum_{j=1}^{N_{\mathrm{M}}-1} \sum_{k=j+1}^{N_{\mathrm{M}}} \sum_{i=1}^{N_{\mathrm{Cl}}} f_{i j} f_{i k}
$$

6.4 Free energy calculations. The 1D-free energy profile for coordination number, $n$, is related to its probability distribution function $(\Omega(n)): W(x)=-k_{\mathrm{B}} T \ln [\Omega(n)]$. 2D-free energy surfaces are also computed using the joint probability distribution function $(\Omega(r, y)): W(r, y)=-k_{\mathrm{B}} T \ln [\Omega(r, y)]$, where $\mathrm{r}$ is the interionic distance and $y$ is the coordination number or number of shared chloride ion between two cations or the number of cations coordinating simultaneously with two different cations via chloride ions. The averaging of the free energies was done considering all possible ion pairs and the production length of the AIMD trajectories.

7. Power spectra calculations. Power spectra revealing various vibrational modes can be calculated by Fourier transforming velocity autocorrelation functions, as given below:

$$
P(\omega)=\int_{0}^{t_{\max }} \frac{<v(t) v(0)>}{<v(0) v(0)>} \cos (\omega t)
$$

In Equation 15, $v$ is the velocity of an ion and $\langle\cdots\rangle$ represents ensemble averaging considering the production length of the AIMD simulations, all ions, and samples starting with an initial time $(t=0)$ and ending with a final time $\left(t_{\max }=1 \mathrm{ps}\right)$, where the correlation function converges to zero.

A power spectrum for the $\mathrm{M}-\mathrm{Cl}$ stretch is calculated using the autocorrelation function of the relative velocities between $\mathrm{Cl}^{-}$and $\mathrm{M}^{2+}\left(v_{\text {rel }}=\frac{d r}{d t}\right.$, where $r$ is the distance between $\mathrm{Cl}^{-}$and $\left.\mathrm{M}^{2+}\right)$ :

$$
P_{\text {Stretch }}(\omega)=\int_{0}^{t_{\mathrm{max}}} \frac{<v_{\text {rel }}(t) v_{\text {rel }}(0)>}{<v_{\text {rel }}(0) v_{\text {rel }}(0)>} \cos (\omega t)
$$

A sample for $P(\omega)$ for a particular coordination state is calculated when that state is found at $t=0$ and assuming that the state remains stable and does not convert to another state until the final time, $t_{\max }$. Here, we chose $t_{\max }=$ $500 \mathrm{fs}$ to ensure that this assumption is reasonable as well as that the velocity autocorrelation function converges to zero at $t_{\max } \cdot\langle\cdots\rangle$ in Equation 16 represents averaging over all $\mathrm{M}-\mathrm{Cl}$ pairs and the samples from the production length of the AIMD simulations. 


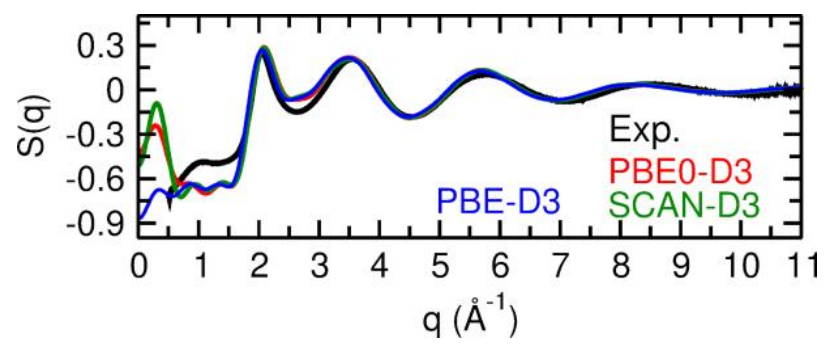

Figure S3. Comparison between the structure functions measured in our X-Ray scattering experiments (at $1123 \mathrm{~K}$ ) and computed from AIMD simulations (at $1073 \mathrm{~K}$ ) using PBE-D3, PBE0-D3, and SCAN-D3 for pure $\mathrm{MgCl}_{2}$.

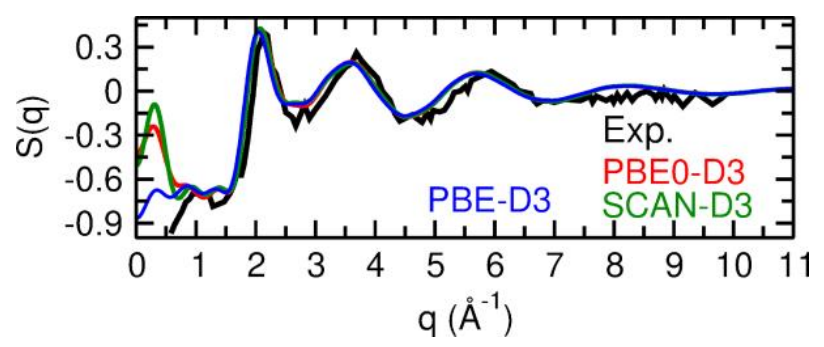

Figure S4. Comparison between the structure functions measured in neutron scattering experiments by Biggin ${ }^{18}$ (at 998 $\mathrm{K}$ ) and computed from AIMD simulations (at $1073 \mathrm{~K}$ ) using PBE-D3, PBE0-D3, and SCAN-D3 for pure $\mathrm{MgCl}_{2}$.

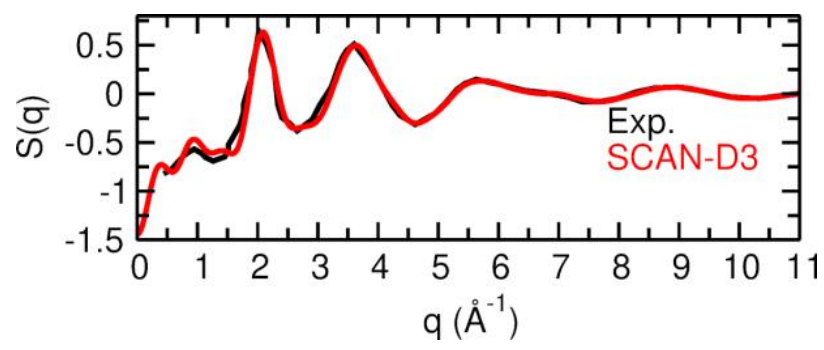

Figure S5. Comparison between the structure functions measured in neutron scattering experiments by Zeidler ${ }^{19}$ (at 605 $\mathrm{K}$ ) and computed from AIMD simulations (at $600 \mathrm{~K}$ ) using SCAN-D3 for pure $\mathrm{ZnCl}_{2}$.

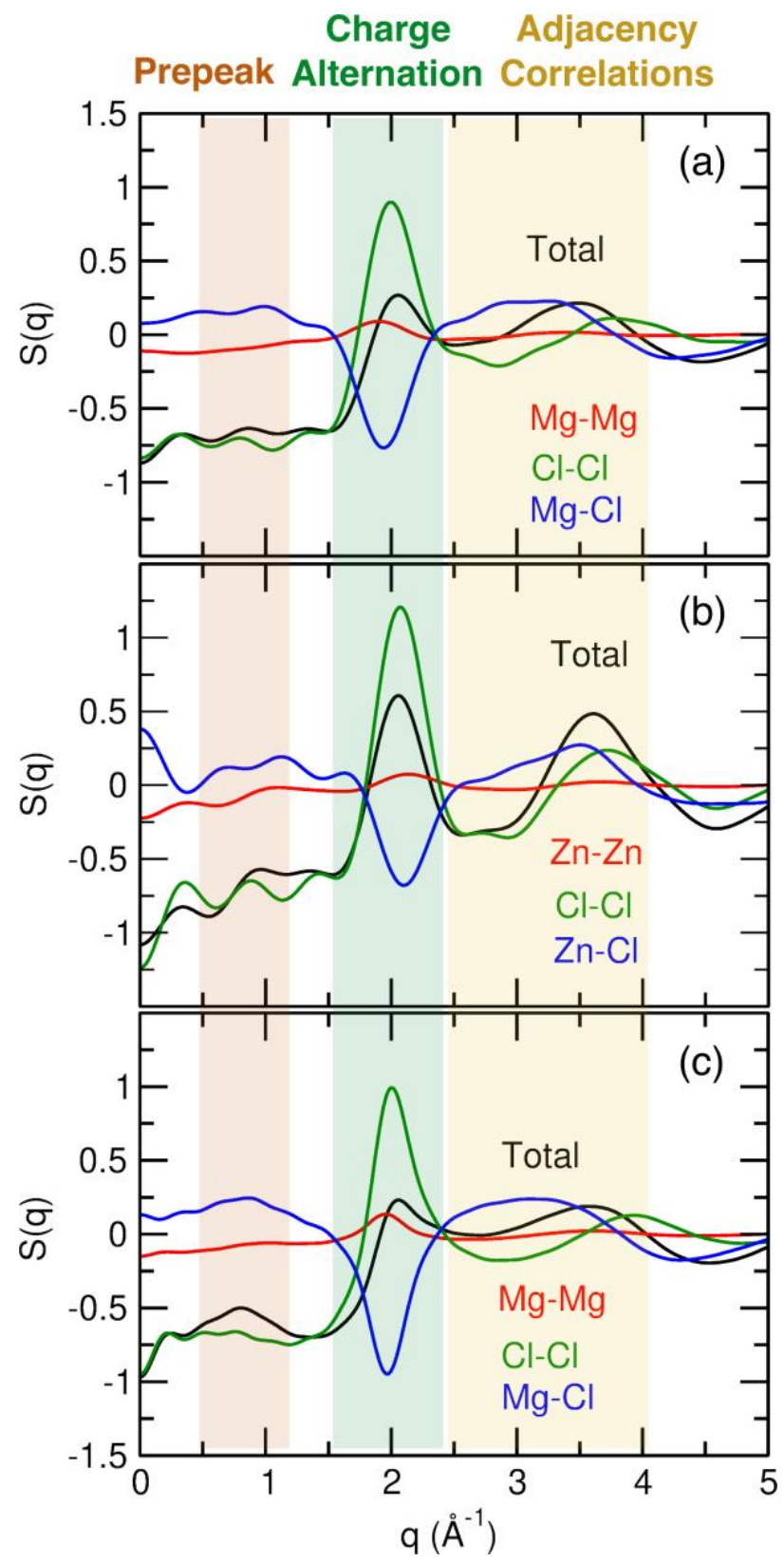

Figure S6. Decompositions of the total structure function into cation-cation, anion-anion, and cation-anion subcomponents (partial structure functions) for (a) pure $\mathrm{MgCl}_{2}$ from the AIMD simulation using PBE-D3 (X-Ray scattering at $1073 \mathrm{~K}$ ), (b) pure $\mathrm{ZnCl}_{2}$ using PBE-D3 (neutron scattering at $600 \mathrm{~K}$ ), and (c) pure $\mathrm{MgCl}_{2}$ using the polarizable ion model and a large simulation box (X-Ray scattering at $1073 \mathrm{~K}$ ) from our previous work,, 30 indicating the existence of adjacency correlation, charge alternation, and intermediate-range ordering reflected in the prepeak (low $q$ ) region. 

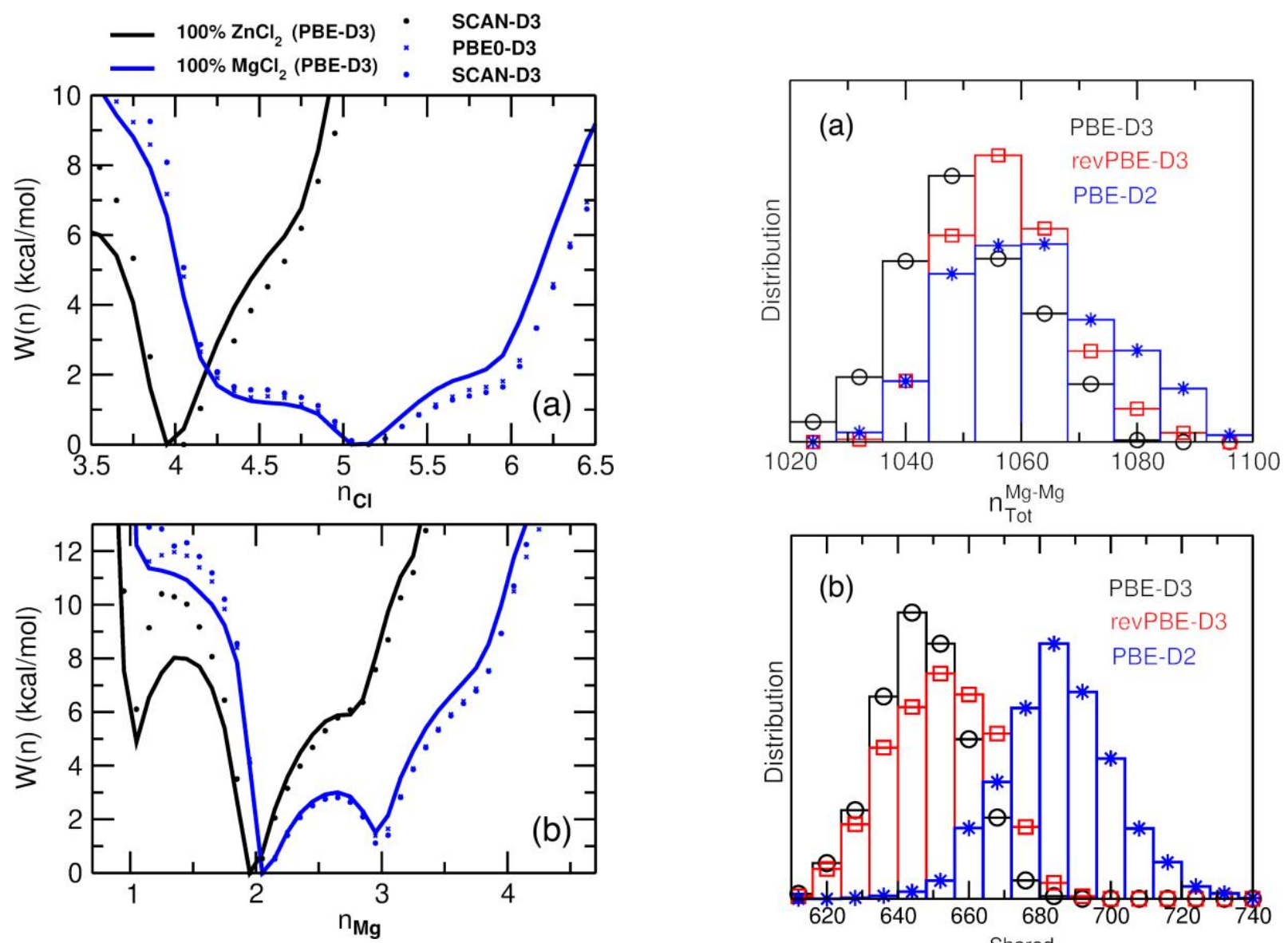

Figure S7. 1D free energy profiles obtained from the AIMD simulations using different DFT functionals for pure $\mathrm{ZnCl}_{2}$ (at $600 \mathrm{~K}$ ) and pure $\mathrm{MgCl}_{2}$ (at $1073 \mathrm{~K}$ ) as functions of the number of $\mathrm{Cl}^{-}$ions around $\mathrm{Mg}^{2+}$ ion (a) and vice versa (b).

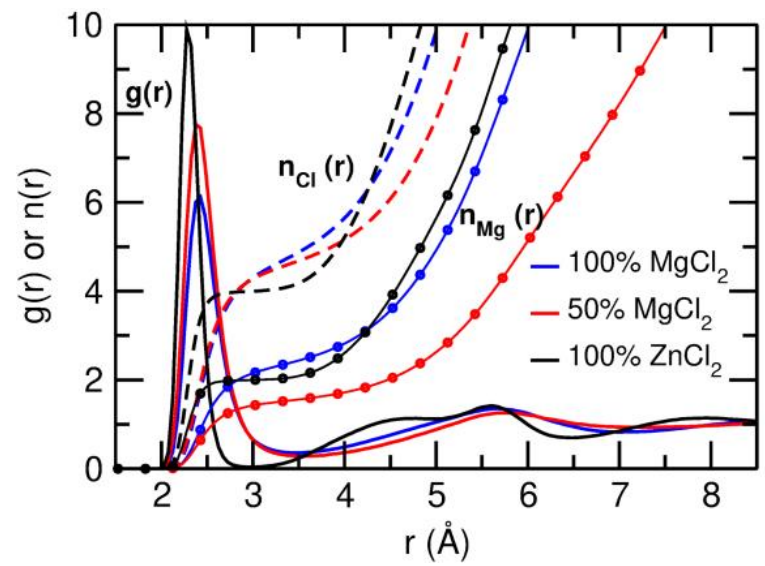

Figure S8. Radial distribution functions and the number of $\mathrm{Cl}^{-}$ ions around a $\mathrm{Mg}^{2+}\left(\mathrm{Zn}^{2+}\right)$ ion (dashed lines) and vice versa (dotted lines) as a function of the $\mathrm{Mg}-\mathrm{Cl}(\mathrm{Zn}-\mathrm{Cl})$ separation distance obtained from the AIMD simulations using PBE-D3 for pure $\mathrm{MgCl}_{2}$ (at $1073 \mathrm{~K}$ ), $50 \%: 50 \% \mathrm{MgCl}_{2}+\mathrm{KCl}$ (at $1073 \mathrm{~K}$ ), and pure $\mathrm{ZnCl}_{2}$ (at $600 \mathrm{~K}$ ). In the pure $\mathrm{ZnCl}_{2}$, the average coordination number of $\mathrm{Zn}^{2+}$ is 4 using the cutoff distance of $3 \AA$. The average coordination of $\mathrm{Mg}^{2+}$ is 4.9 in pure $\mathrm{MgCl}_{2}$ and 4.7 in the mixture using the cutoff distance of $3.5 \AA$. Using the same cutoff distances the coordination number of $\mathrm{Cl}^{-}$is 2.0 in pure $\mathrm{ZnCl}_{2}, 2.4$ in pure $\mathrm{MgCl}_{2}$, and 1.5 in $50 \%: 50 \% \mathrm{MgCl}_{2}+\mathrm{KCl}$.
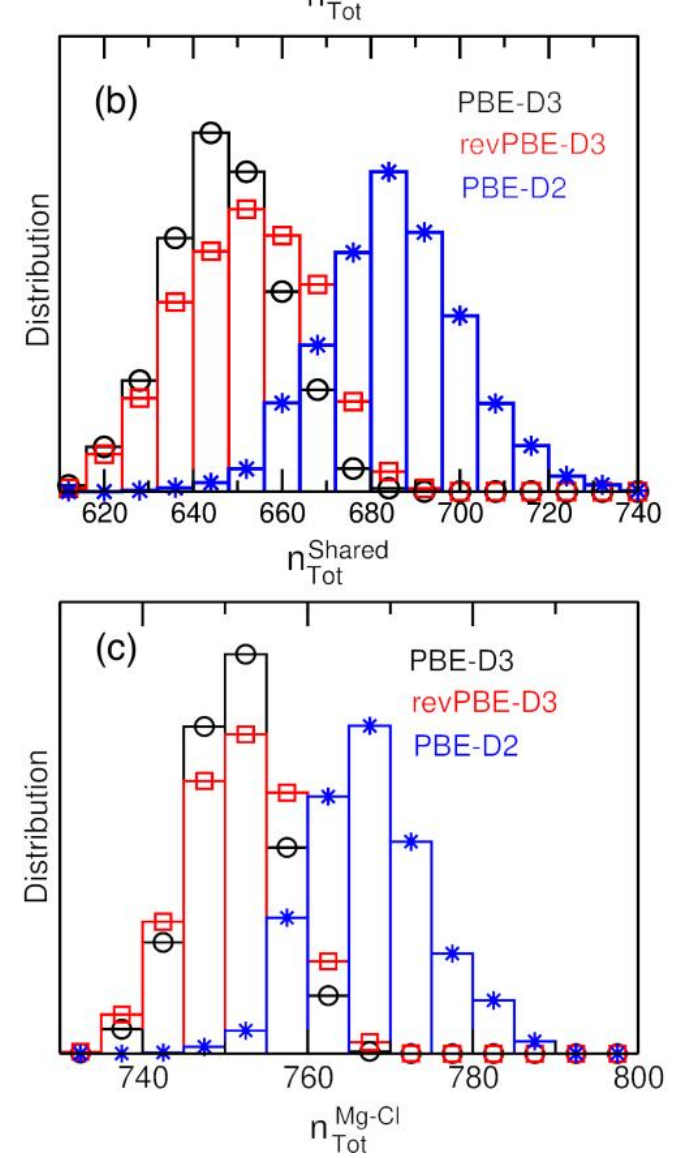

Figure S9. Histograms of the total number of $\mathrm{Mg}-\mathrm{Mg}$ contacts $\left(n_{\text {Tot }}^{M g-M g}\right)(\mathrm{a})$, the total number of shared chloride ions $\left(n_{\text {Tot }}^{\text {Shared }}\right)$ (b), and the total number of $\mathrm{Mg}-\mathrm{Cl}$ contacts $\left(n_{T o t}^{M g-C l}\right)$ (c) obtained from the AIMD simulations using different DFT functionals for pure $\mathrm{MgCl}_{2}$ at $1073 \mathrm{~K}$. 


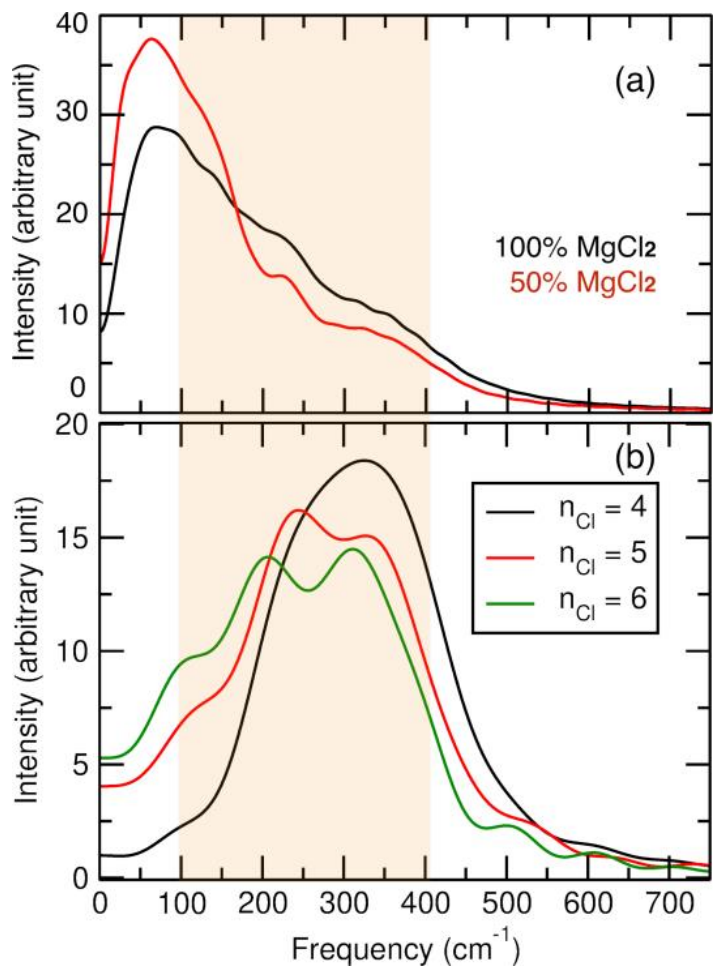

Figure S10. Power spectra of pure $\mathrm{MgCl}_{2}$ and for the 50\%:50\% $\mathrm{MgCl}_{2}+\mathrm{KCl}$ mixture at $1073 \mathrm{~K}$ using the PBE-D3 density functional (a). Power spectra for the $\mathrm{M}-\mathrm{Cl}$ stretch in different coordination states of $\mathrm{Mg}^{2+}$ in the $50 \%: 50 \% \mathrm{MgCl}_{2}+\mathrm{KCl}$ mixture at the same temperature and using the same DFT functional (b). The highlighted region indicates the frequency range for which the Raman spectra are reported in this paper.

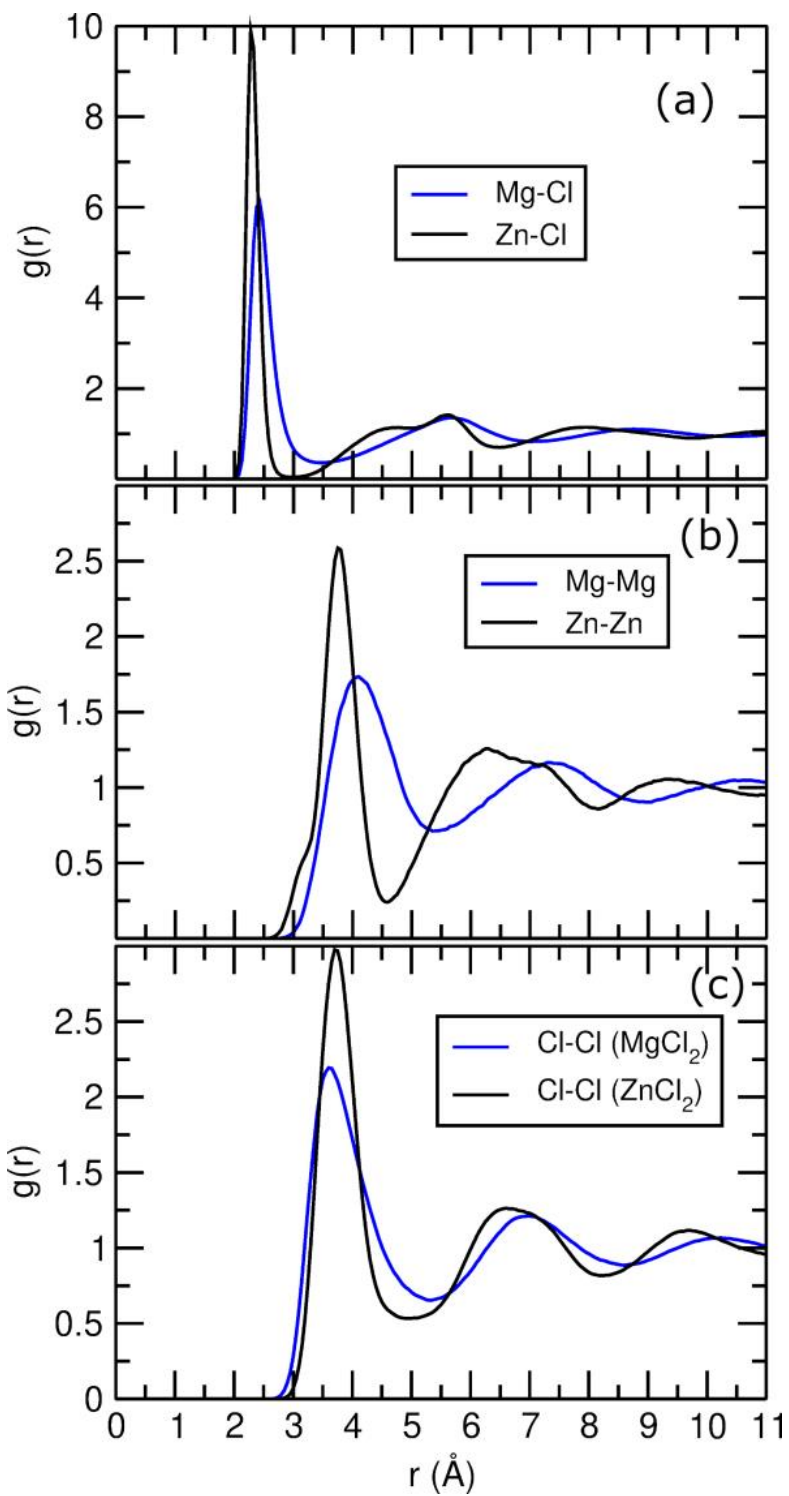

Figure S11. Radial distribution functions for cation-anion (a), cation-cation (b), and anion-anion (c) distances obtained from the AIMD (PBE-D3) simulations of pure $\mathrm{MgCl}_{2}$ (at $1073 \mathrm{~K}$ ) and pure $\mathrm{ZnCl}_{2}$ (at $600 \mathrm{~K}$ ), which are used to assign the most probable close-contact distances listed in Table S1. 


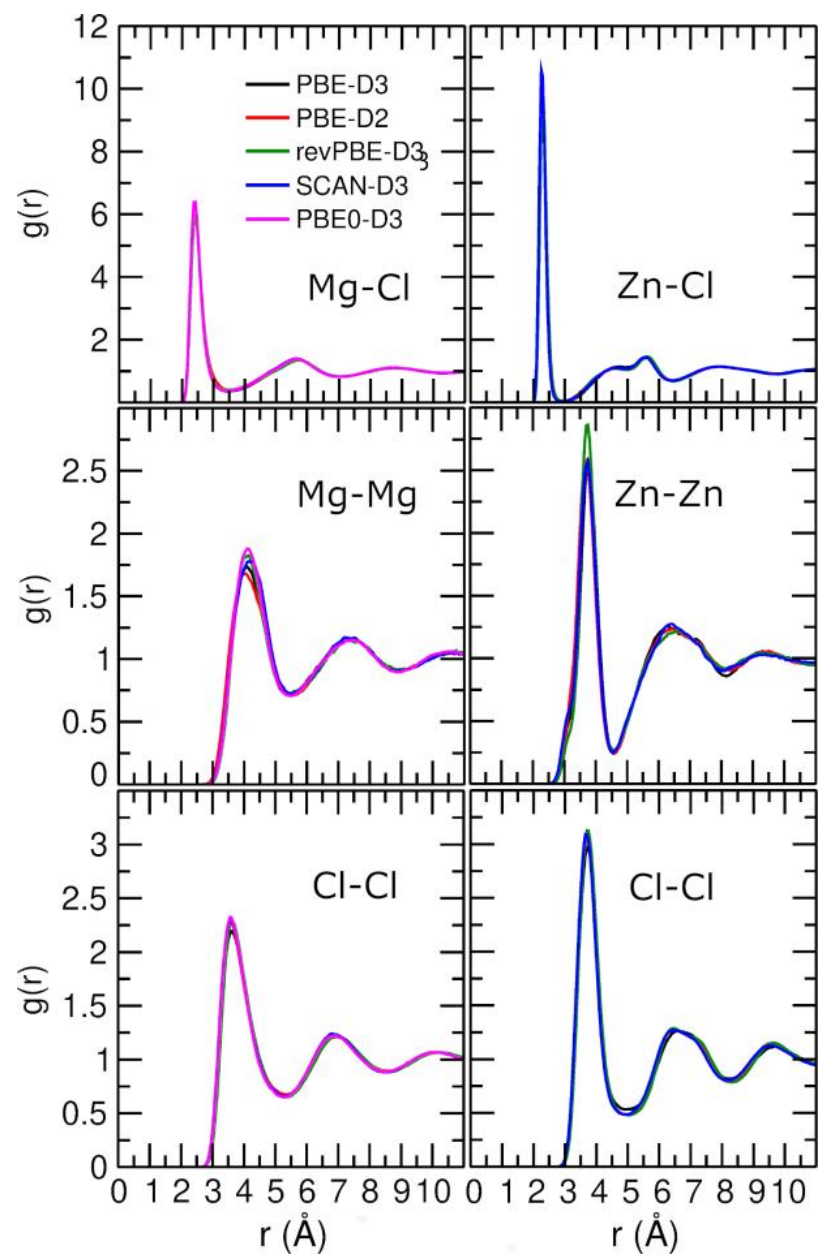

Figure S12. Radial distribution functions for cation-anion (top panel), cation-cation (middle panel), and anion-anion (bottom panel) distances obtained from the AIMD simulations of pure $\mathrm{MgCl}_{2}$ (at $1073 \mathrm{~K}$ ) and pure $\mathrm{ZnCl}_{2}(600 \mathrm{~K})$ using different DFT functionals, highlighting their ability to predict the same equilibrium interionic distances.

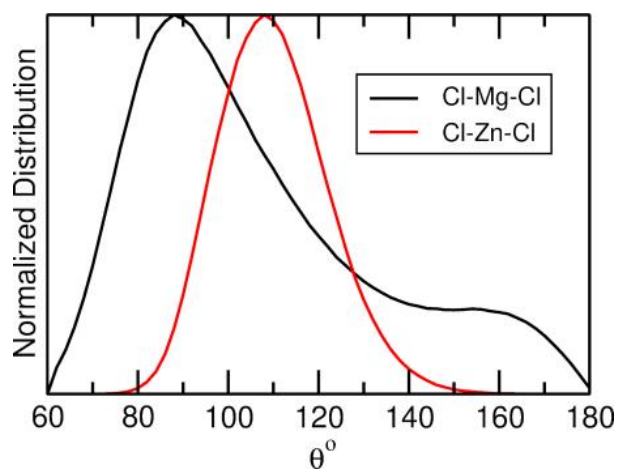

Figure S13. Histograms of anion-cation-anion angles in pure $\mathrm{MgCl}_{2}$ and pure $\mathrm{ZnCl}_{2}$, considering all coordination states of $\mathrm{Mg}^{2+}$ and $\mathrm{Zn}^{2+}$ computed using the PBE-D3 functional.

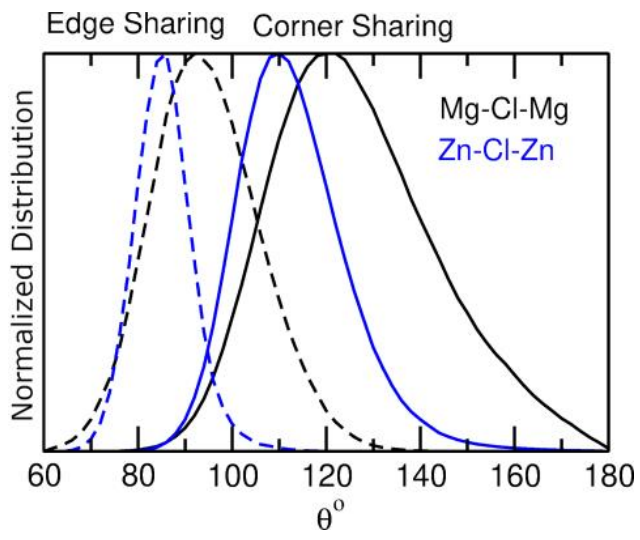

Figure S14. Histograms of cation-anion-cation angles in pure $\mathrm{MgCl}_{2}$ (at $1073 \mathrm{~K}$ ) and pure $\mathrm{ZnCl}_{2}$ (at $600 \mathrm{~K}$ ) using the PBE-D3 functional for the edge-shared (dashed line; two $\mathrm{Cl}^{-}$shared between two cations) and corner-shared (solid line; one $\mathrm{Cl}^{-}$ shared between two cations) structures. 


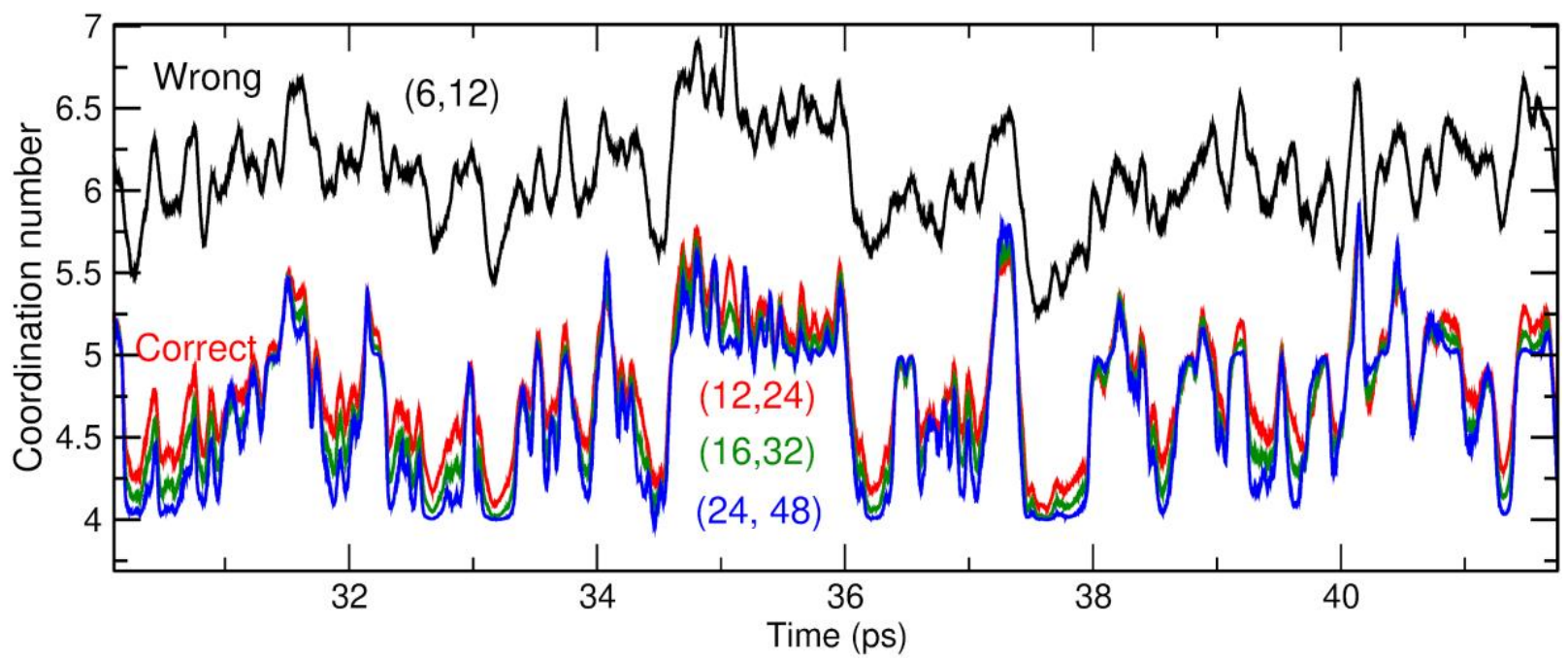

Figure S15. Coordination number as a function of time (for $\mathrm{Mg}^{2+}$ in pure $\mathrm{MgCl}_{2}$ at $800{ }^{\circ} \mathrm{C}$ using PBE-D3): Choice of powers in Equation 10 cannot be as low as $(6,12)$ as they will provide wrong, overestimated coordination numbers, but power like $(12,24),(16,32),(24,48)$ are acceptable - the latter two provide sharper transitions

Table S1. Equilibrium cation (M)-cation $(\mathrm{M})$ and anion $(\mathrm{Cl})$ anion $(\mathrm{Cl})$ close-contact distances in pure molten $\mathrm{MgCl}_{2}(\mathrm{~T}=$ $1073 \mathrm{~K})$ and $\mathrm{ZnCl}_{2}(\mathrm{~T}=600 \mathrm{~K})$, obtained from the first peak of the RDFs computed from the AIMD-PBE-D3 trajectories (Figures S11 and S12), which are the same as those obtained using other DFT functionals. Error bars were determined from the half-width (full width at half of the maximum) of the RDFs. The values for the $\mathrm{M}-\mathrm{Cl}$ distances for different coordination states are assigned from the histogram in Figure $7 \mathrm{a}$ of the main text. Angles are assigned from the histograms in Figures $7 \mathrm{~b}$ and $7 \mathrm{c}$.

\begin{tabular}{|l|l|l|}
\hline & $\mathrm{MgCl}_{2}$ & $\mathrm{ZnCl}_{2}$ \\
\hline $\begin{array}{l}\text { Distance } \\
(\mathrm{A})\end{array}$ & & \\
\hline $\mathrm{M}-\mathrm{M}$ & $4.10 \pm 0.75$ & $3.75 \pm 0.35$ \\
$\mathrm{Cl}-\mathrm{Cl}$ & $3.61 \pm 0.60$ & $3.71 \pm 0.36$ \\
& & \\
$\mathrm{M}-\mathrm{Cl}$ & $2.39 \pm 0.17(\mathrm{n}=4)$ & $2.30 \pm 0.12(\mathrm{n}=4)$ \\
& $\begin{array}{l}2.44 \pm 0.22(\mathrm{n}=5) \\
\end{array}$ & $2.49 \pm 0.28(\mathrm{n}=6)$ \\
& $\mathrm{RDF})$ & $2.30 \pm 0.20(\mathrm{from}$ \\
& & $(\mathrm{from} \mathrm{RDF})$ \\
\hline & & \\
Angle & $102^{\circ} \pm 21^{\circ}(\mathrm{n}=4)$ & $108^{\circ} \pm 15^{\circ}(\mathrm{n}=4)$ \\
& $88^{\circ} \pm 17^{\circ}(\mathrm{n}=5)$ & \\
\hline $\mathrm{Cl}-\mathrm{M}-\mathrm{Cl}$ & $96^{\circ} \pm 27^{\circ}$ & $110^{\circ} \pm 12^{\circ}$ \\
\hline
\end{tabular}

\section{REFERENCES}

1. Kurley, J.; Halstenberg, P.; McAlister, A.; Raiman, S.; Dai, S.; Mayes, R., Enabling chloride salts for thermal energy storage: implications of salt purity. Rsc Advances 2019, 9 (44), 25602-25608.

2. Kipouros, G. J.; Sadoway, D. R., A thermochemical analysis of the production of anhydrous $\mathrm{MgCl}_{2}$. Journal of Light Metals 2001, 1, 111-117.

3. $\quad \mathrm{Wu}$, F.; Roy, S.; Ivanov, A.; Gill, S.; Topsakal, M.; Dooryhee, E.; Abeykoon, M.; Kwon, G.; Gallington, L.; Halstenberg, P. et al. Elucidating Ionic Correlations Beyond Simple Charge Alternation in Molten $\mathrm{MgCl}_{2}-\mathrm{KCI}$ Mixtures. Journal of Physical Chemistry Letters 2019, 10 (24), 7603-7610. 4. Toby, B.; Von Dreele, R., GSAS-II: the genesis of a modern open-source all purpose crystallography software package. Journal of Applied Crystallography 2013, 46, 544-549. 5. Qiu, X.; Thompson, J. W.; Billinge, S. J. L., PDFget X2 : a GUI-driven program to obtain the pair distribution function from X-ray powder diffraction data. Journal of Applied Crystallography 2004, 37, 678-678.

6. $\quad$ Egami, T.; Billinge, S. J., Underneath the Bragg peaks: structural analysis of complex materials. 2nd ed.; ELSEVIER: 2012; Vol. 16.

7. Fischer, H.; Barnes, A.; Salmon, P., Neutron and x-ray diffraction studies of liquids and glasses. Reports on Progress in Physics 2006, 69 (1), 233-299.

8. Dai, S.; Begun, G.; Young, J.; Mamantov, G., Application of chemometric methods in raman-spectroscopic studies of molten-salt system containing $\mathrm{MgCl}_{2}-\mathrm{KCl}$ experimental-evidence for existence of $\mathrm{Mg}_{2} \mathrm{Cl}_{7}^{3-}$ dimer and its raman-spectrum. Journal of Raman Spectroscopy 1995, 26 (10), 929-932.

9. Huang, C.; Brooker, M., Raman-spectrum of molten $\mathrm{MgCl}_{2}$. Chemical Physics Letters 1976, 43 (1), 180-182.

10. Alsayoud, A.; Rao, M.; Edwards, A.; Deymier, P.; Muralidharan, K.; Potter, B.; Runge, K.; Lucas, P., Structure of $\mathrm{ZnCl}_{2}$ Melt. Part I: Raman Spectroscopy Analysis Driven by $\mathrm{Ab}$ Initio Methods. Journal of Physical Chemistry B 2016, 120 (17), 4174-4181. 
11. Brown P.J., F. A. G., Maslen E.N., O'Keefe M.A., Willis B.T.M., Intensity of diffracted intensities, International Tables for Crystallography. Springer: 2006.

12. Sears, V. F., Neutron scattering lengths and cross sections. Neutron News 1992, 3, 26-37.

13. Putrino, A.; Parrinello, M., Anharmonic Raman spectra in high-pressure ice from ab initio simulations. Physical Review Letters 2002, 88 (17).

14. Thomas, M.; Brehm, M.; Fligg, R.; Vohringer, P.; Kirchner, B., Computing vibrational spectra from ab initio molecular dynamics. Physical Chemistry Chemical Physics 2013, 15 (18), 6608-6622.

15. Brehm, M.; Kirchner, B., TRAVIS - A Free Analyzer and Visualizer for Monte Carlo and Molecular Dynamics Trajectories. Journal of Chemical Information and Modeling 2011, 51 (8), 2007-2023.

16. Brehm, M.; Thomas, M.; Gehrke, S.; Kirchner, B., TRAVIS-A free analyzer for trajectories from molecular simulation. Journal of Chemical Physics 2020, 152 (16).
17. Brehm, M.; Thomas, M., Computing Bulk Phase Raman Optical Activity Spectra from ab initio Molecular Dynamics Simulations. Journal of Physical Chemistry Letters 2017, 8 (14), 3409-3414.

18. Biggin, S.; Gay, M.; Enderby, J., The structures of molten magnesium and manganese(ii) chlorides. Journal of Physics C-Solid State Physics 1984, 17 (6), 977-985.

19. Zeidler, A.; Salmon, P.; Martin, R.; Usuki, T.; Mason, P.; Cuello, G.; Kohara, S.; Fischer, H., Structure of liquid and glassy $\mathrm{ZnCl}_{2}$. Physical Review B 2010, 82 (10).

20. Wu, F.; Sharma, S.; Roy, S.; Halstenberg, P.; Gallington, L.; Mahurin, S.; Dai, S.; Bryantsev, V.; Ivanov, A.; Margulis, C., Temperature Dependence of Short and Intermediate Range Order in Molten $\mathrm{MgCl}_{2}$ and Its Mixture with $\mathrm{KCl}$. Journal of Physical Chemistry B 2020, 124 (14), 2892-2899. 